\title{
Nível de conhecimento da simbologia gráfica utilizada para caracterizar comandos e controles de máquinas agrícolas
}

\author{
Level of knowledge of graphic symbols used to characterize commands and controls of \\ agricultural machines
}

\author{
Airton dos Santos Alonço ${ }^{\mathrm{I}}$ Antônio Lilles Tavares Machado ${ }^{\mathrm{II}}$ \\ Mauro Fernando Pranke Ferreira ${ }^{\text {III }}$ \\ Fabrício Ardais Medeiros ${ }^{\mathrm{IV}}$
}

\section{RESUMO}

Este trabalho foi realizado no período compreendido entre março de 2000 a junho de 2005, com entrevistados de várias regiões do Brasil, e teve por objetivo realizar uma pesquisa analítica a respeito do grau de conhecimento sobre símbolos gráficos, para a identificação dos comandos e controles de operação e manutenção em máquinas agrícolas, dos principais clientes envolvidos no processo, desde o projeto da máquina agrícola até sua utilização e conseqüente manutenção. Os resultados encontrados indicam que o conhecimento sobre o significado que possuem os símbolos gráficos utilizados para caracterizar comandos e controles de máquinas agrícolas é bastante reduzido por parte dos principais envolvidos no processo.

Palavras-chave: normas técnicas, engenharia agrícola, máquinas agrícolas, segurança, ergonomia.

\section{ABSTRACT}

The present study was carried out from March 2000 to June 2005 by interviewing many people from several parts of Brazil. It was aimed at assessing the level of knowledge on technical graphic symbols used for identification of commands and operation controls, and support in agricultural machines. The main customers involved in the process from the project of the agricultural machine to its use and support were interviewed. Results suggest that the knowledge of the graphic symbols meaning is quite reduced among the main customers involved in the process.

Key words: graphic symbols, standards, agricultural engineering, agricultural machines, safety, ergonomics.

\section{INTRODUÇÃO}

A mente humana funciona como um sistema de órgãos de computação, desenhado por seleção natural para resolver os tipos de problemas que nossos ancestrais encaravam nas suas maneiras de viver (PINKER, 1998). Assim sendo, cada módulo ou órgão mental apresenta um "design" especializado que o faz especialista em algum tipo de interação com o mundo. A lógica básica dos módulos é especificada pelo programa genético e a forma como operam foi conformada pela seleção natural para resolver problemas da vida de caça e de coleta das sociedades primitivas.

O organismo humano dispõe de cerca de um bilhão de receptores (FIALHO, 2000). De cada órgão dos sentidos, originam-se seqüências de impulsos que passam por vários milhões de condutos nervosos em direção ao sistema nervoso central. Cada um desses condutos é capaz de transmitir informações a uma velocidade 10 a $100 \mathrm{bits} \mathrm{seg}^{-1}$, de modo que a entrada máxima de informações é de 10 a 100 milhões de bits por segundo. Desse total, apenas uma ínfima fração, cerca de 25bits por segundo, atinge a consciência. Portanto, como é possível observar na figura 1, o ser humano recebe cerca de $10^{9}$ bits por segundo de informação provenientes do mundo exterior, dos quais

'Programa de Pós-graduação em Engenharia Agrícola, Departamento de Engenharia Rural, Centro de Ciências Rurais (CCR), Universidade Federal de Santa Maria (UFSM), 97105-900, Santa Maria, RS, Brasil. E-mail: alonço@ccr.ufsm.br. Autor para correspondência.

"Departamento de Engenharia Rural, Faculdade de Agronomia "Eliseu Maciel”, Universidade Federal de Pelotas (UFPel), Pelotas, RS, Brasil. E - mail: lilles@ufpel.edu.br.

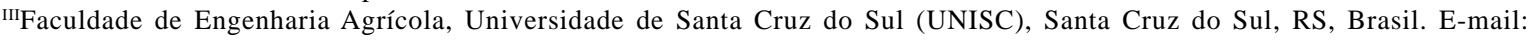
maurofernandoferreira@yahoo.com.br.

IvPrograma de Pós-graduação em Engenharia Agrícola, UFSM, Santa Maria, RS, Brasil. E-mail: medeiros_f@terra.com.br. 


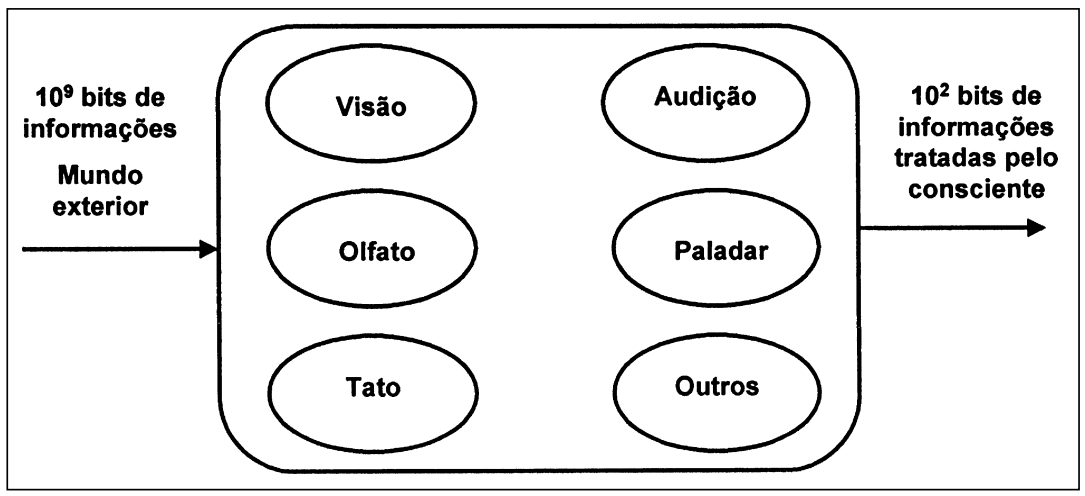

Figura 1 - Processamento das informações vindas do mundo exterior. Fonte: Fialho, 2000
A noção de MCT foi progressivamente abandonada, dando lugar à “Memória de Trabalho"(MT). A diferença é que esta última é concebida como um sistema que realiza não só o armazenamento, mas também o tratamento das informações, c a racterística ma is fundamental e que requer mais investimento para a segurança.

Por outro lado, as
$10^{2}$ bits por segundo de informação são processados a um nível consciente.

Dentro da Modelagem Cognitiva, sensação é a resposta específica a um estímulo sensorial particular, enquanto percepção é o conjunto de mecanismos de codificação e de coordenação das diferentes sensações elementares, visando a um significado.

O sistema sensitivo humano clássico compreende a visão, a audição, o tato, o paladar e o olfato. As características da informação conservada nos “registros sensoriais”, segundo ALONÇO (2004), são as seguintes: a informação conservada é uma imagem precisa do estímulo tal como ele é percebido pelo órgão sensorial; esta informação não é decodificada, mas bruta, não sendo modificada por mecanismos cognitivos; a duração deste primeiro registro corresponde a menos de um segundo para sensações visuais e a alguns segundos para as auditivas; ela é volátil, qualquer que seja a vontade do sujeito que não pode mantê-la, mesmo por um esforço de auto-repetição mental; a capacidade do registro sensorial é limitada, dependendo do tipo de órgão sensorial envolvido; em função da rápida queda da informação no registro sensorial, somente parte dela é selecionada ou filtrada para processamento posterior.

Na "Memória de Curto Termo" (MCT), a informação conservada é totalmente diferente: a informação já é o resultado de uma interpretação; pode ser síntese de várias informações provenientes de diferentes registros sensoriais (por exemplo: visão + audição), mas não retém a totalidade das informações; a capacidade desta memória é limitada e volátil; conserva a informação durante segundos; pode ser mantida, mas fica extremamente sensível às interferências de outras informações (exemplo disso é quando repete-se um número de telefone e alguém perturba com uma pergunta) (ALONÇO, 2004). características mais importantes da "Memória de Longo Termo” (MLT) são: organização mais complexa, não apresentando um declínio sistemático em função do tempo; capacidade praticamente ilimitada (isto não impede o esquecimento que se dá não por razões de capacidade, mas de organização ou de mecanismos de decodificação); dependência, primeiramente, das transformações operadas por filtragem da memória de curto termo para, em seguida, integrar a informação às estruturas que compõem os esquemas cognitivos e às informações já memorizadas; conservação das informações de forma permanente (ALONÇO, 2004).

Durante muitos anos, o desenvolvimento em automatização e/ou automação, os sistemas de apoio ao operador de máquinas e as políticas de segurança foram feitos com o propósito de suprimir ou prevenir o erro humano (KONTOGIANNIS, 1999). A política de "acidente zero", como sendo a última meta de segurança, foi discutida e tentada exaustivamente, porém esta confiança exclusiva na "supressão do erro" tem sido questionada por vários pesquisadores, como FRESE, (1991), ZAPF et al., (1994) e CARPES JÚNIOR (2001), até mesmo em sistemas tecnologicamente avançados, pois funcionamentos inadequados de sistemas de segurança e más adaptações para as interações com o usuário, freqüentemente, resultaram em sérios acidentes. Além disso, sempre é previsto que os operadores atuem com eficiência em situações inesperadas, em que seja necessário desenvolver estratégias complexas e que usem seus conhecimentos sob constrangimentos de tempo exíguo e tensão psicológica.

As atividades de projeto podem estar sujeitas a deslizes ou enganos, serem incorretas ou impróprias. Isto sugere então que os erros possam se agrupar de acordo com o local onde acontecem e da forma como acontecem.

Ao classificarem onde podem acontecer erros em informação relativos a um determinado produto, McMAHON et al.(1997), entre outros autores, 
afirmam que referências incorretas em normas técnicas ou em códigos podem causar sérios erros em relação às exigências funcionais do produto e que valores de normas técnicas incorretamente transcritos podem influenciar negativamente valores de alguns atributos implícitos do produto.

Em seu estudo sobre normas técnicas para o projeto de produtos, MUCKLER (1984) afirma que todas as decisões de projeto sobre controles e comandos são extremamente dependentes da profundidade e da precisão da análise da tarefa a ser executada o que por sua vez, também o são para a confecção das normas técnicas que definem os símbolos gráficos utilizados para a identificação dos comandos e controladores de máquinas agrícolas.

No Brasil, a ABNT (1990), totalmente baseada na composição da ASAE S304.5 (1984), do ISO 3767/1 (1982) e do ISO 3767/2 (1982), publicou a NBR 11379 - Símbolos gráficos para máquinas agrícolas, com o objetivo de padronizar o uso dos símbolos gráficos para identificação dos comandos de operação e manutenção em máquinas agrícolas.
Desta forma, levando em consideração os aspectos anteriormente definidos por MUCKLER (1984), GIBBINGS (1986), McMAHON et. al. (1997), PINKER (1998), KONTOGIANNIS (1999), FIALHO (2000) eALONÇO (2004), este trabalho teve por objetivo realizar uma pesquisa analítica a respeito do grau de conhecimento sobre símbolos gráficos para a identificação dos comandos e controles de operação e de manutenção em máquinas agrícolas, dos principais clientes envolvidos no processo, desde o projeto da máquina agrícola até sua utilização e conseqüente manutenção.

\section{MATERIAL E MÉTODOS}

Este trabalho foi realizado no período compreendido entre março de 2000 a junho de 2005 e constituiu-se na aplicação de um questionário (Figura 2), no que foi solicitado ao entrevistado que dissesse o significado de cada símbolo perguntado.

Este questionário foi composto por 20\% (1/ 5) da totalidade dos símbolos contidos na NBR 11379

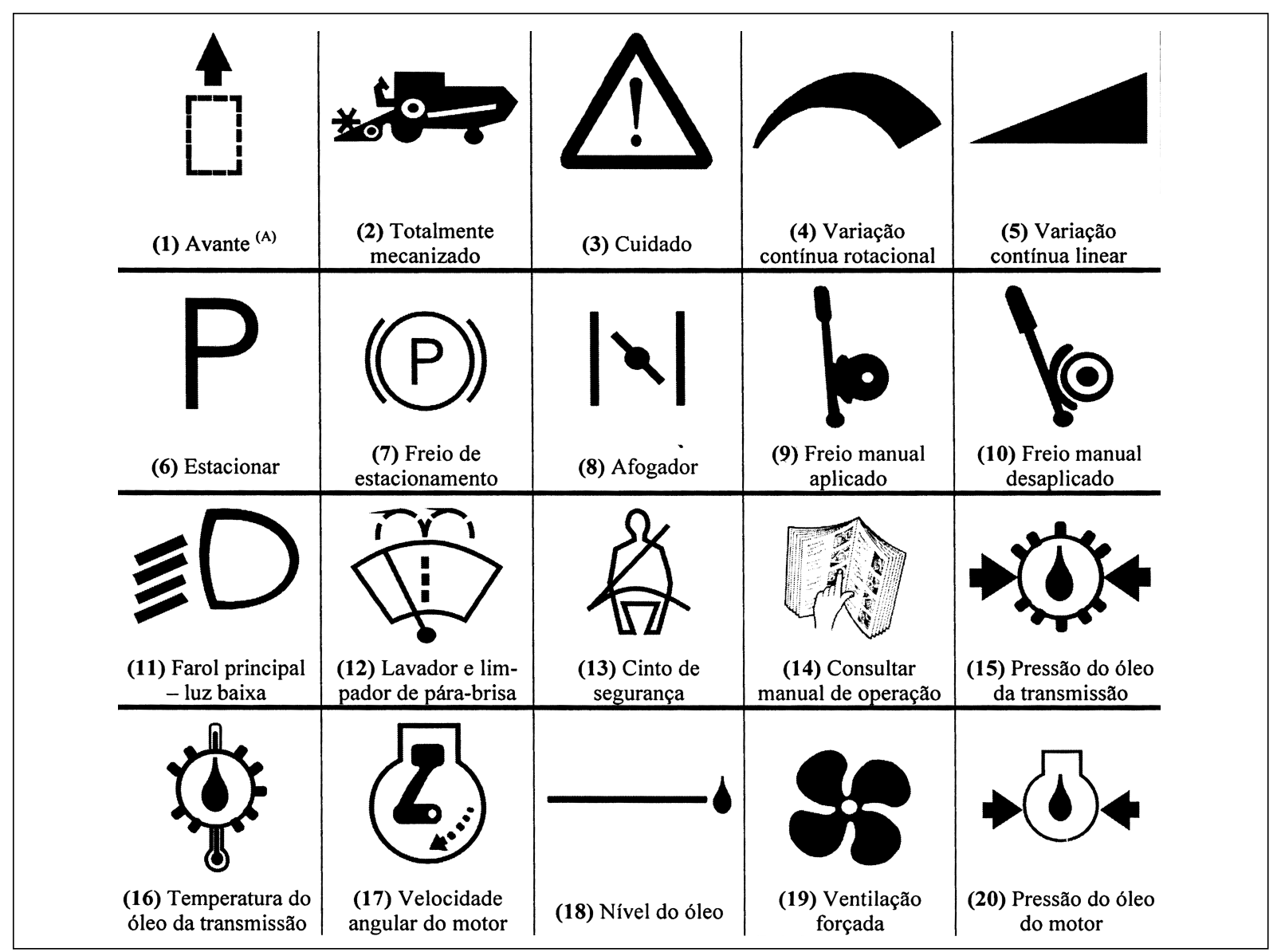

Figura 2 - Questionário aplicado aos clientes de máquinas agrícolas e significado dos símbolos sorteados. Fonte: ABNT, 1990.

Ciência Rural, v.37, n.1, jan-fev, 2007. 
- Símbolos gráficos para máquinas agrícolas (ABNT, 1990), retirados ao acaso, compondo assim uma amostra representativa, de acordo com as recomendações para a composição de uma amostra, pois, conforme SPIEGEL (1993), "a fim de que as conclusões da teoria de amostragem e da inferência estatística sejam válidas, as amostras deverão ser escolhidas de modo a serem representativas de uma população". O mesmo foi aplicado em três distintas categorias:

a) operadores/mantenedores: categoria composta por operadores de máquinas agrícolas profissionais e por mecânicos especialistas neste tipo de equipamento. Nesta categoria, foram entrevistados cento e oitenta e dois (182) profissionais de todas as regiões do Brasil que estivessem participando de feiras agropecuárias, como a, Agrishow (Ribeirão Preto - SP) e a Expodireto (Não-me-Toque-RS), e também em propriedades rurais de todo o país e em algumas da Argentina e do Uruguai, que foram visitadas pelos autores;

b) profissionais: categoria constituída por engenheiros agrícolas, engenheiros florestais, engenheiros agrônomos e engenheiros mecânicos, em sua grande maioria possuidores de títulos de mestre e/ou de doutor e atuantes em ensino, pesquisa, projeto e desenvolvimento na área de máquinas e mecanização agrícola. Nesta categoria, foram entrevistados setenta e dois (72) profissionais oriundos de várias instituições de ensino do Brasil e do exterior, como: UFPA(Belém PA); FURG (Rio Grande - RS); UNB (Brasília - DF); UFLA (Lavras-MG); UFSM (Santa Maria-RS); UFES (Vitória - ES); UFMT (Cuiabá - MT); UFRGS (Porto Alegre-RS); USP (São Paulo-SP); UNESP (Jaboticabal e Botucatu - SP); UNICAMP (Campinas - SP); UFPel (Pelotas - RS); UNIJUI (Ijuí - RS); ULBRA (Canoas RS); UPM (Madri - Espanha); UFSC (Florianópolis SC); UDESC (Florianópolis, Lajes e Joinville - SC); UNISC (Santa Cruz do Sul - RS); UFV (Viçosa - MG); UCS (Caxias do Sul - RS); ESALQ (Piracicaba - SP); UEM (Maringá-PR); UFGO (Goiânia-GO); UFSCAR (São Carlos - SP); UFCG (Campina Grande - PB); UFPR (Curitiba - PR); UFRPE (Recife - PE); UPF
(Passo Fundo - RS); UNICRUZ (Cruz Alta - RS) e outras;

c) acadêmicos: categoria constituída por alunos do sétimo semestre em diante dos cursos de Engenharia Agrícola, Agronomia, Engenharia Florestal e Engenharia Mecânica existentes na UFSM (Santa Maria - RS), UFPel (Pelotas - RS), UFSC (Florianópolis) e UNISC (Santa Cruz do Sul-RS) (Total de entrevistados: 164).

Após a coleta das informações, foi estabelecido como critério de classificação o percentual de acertos obtidos em cada uma das cinco classes préestabelecidas ( 0 - 3,9; 4,0 - 7,9; 8,0 - 11,9; 12,0 - 15,9; 16,0 - 20), para cada categoria entrevistada.

Para a análise dos dados, foi aplicado o teste $\mathrm{Z}$ para comparação entre duas proporções, entre as categorias em cada classe de acertos estabelecidos. Nas análises estatísticas adotou-se 5\% de probabilidade de erro.

\section{RESULTADOS E DISCUSSÃO}

Os resultados obtidos sobre os valores absolutos (Tabela 1) e percentuais (Figura 3) dos acertos de cada categoria entrevistada demonstram o quão despreparados encontram-se todos os entrevistados, independentemente da categoria à qual pertencem. Porém, na classe de acertos compreendida entre 4 e 7,9, de forma positiva, a categoria dos acadêmicos diferencia-se estatisticamente. Por outro lado, na classe de acertos compreendida entre 0 e 3,9 acertos, também de forma positiva, a categoria dos operadores/mantenedores diferencia-se estatisticamente.

Essas afirmações anteriores e o fato de que, em todas as outras classes de acertos, o percentual é extremamente baixo indicam a necessidade urgente de este tema ser abordado em cursos universitários regulares e em cursos de atualização dirigidos para os operadores/mantenedores e profissionais, como forma de capacitar os futuros responsáveis pelo projeto de

Tabela 1 - Valores absolutos e percentuais dos acertos de cada categoria entrevistada.

\begin{tabular}{|c|c|c|c|c|c|c|c|c|c|c|}
\hline & \multicolumn{10}{|c|}{ Número e percentual de acertos } \\
\hline & \multicolumn{2}{|c|}{ De 0,0 a 3,9} & \multicolumn{2}{|c|}{ De 4,0 a 7,9} & \multicolumn{2}{|c|}{ De 8,0 a 11,9} & \multicolumn{2}{|c|}{ De 12,0 a 15,9} & \multicolumn{2}{|c|}{ De 16,0 a 20,0} \\
\hline & \multicolumn{2}{|c|}{ Total } & \multicolumn{2}{|c|}{ Total } & \multicolumn{2}{|c|}{ Total } & \multicolumn{2}{|c|}{ Total } & \multicolumn{2}{|c|}{ Total } \\
\hline Acadêmicos & 20 & b & 92 & a & 47 & $\mathrm{a}$ & 5 & a & 0 & $\mathrm{a}$ \\
\hline Profissionais & 6 & b & 24 & $\mathrm{~b}$ & 28 & $\mathrm{a}$ & 12 & a & 2 & $\mathrm{a}$ \\
\hline Oper./mant. & 64 & a & 67 & $\mathrm{~b}$ & 48 & $\mathrm{a}$ & 3 & a & 0 & $\mathrm{a}$ \\
\hline
\end{tabular}

*Proporções não seguidas pela mesma letra na coluna diferem pelo teste Z a 5\% de significância. 


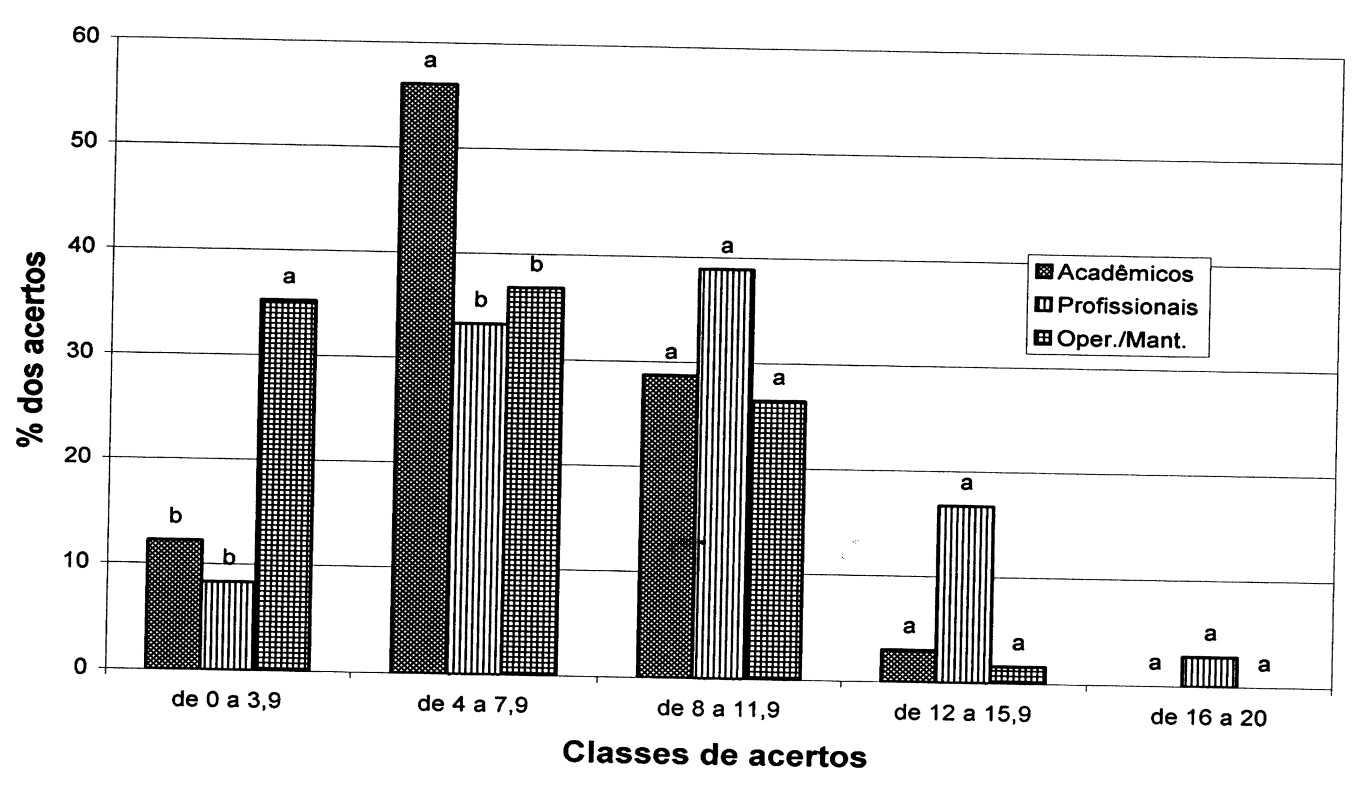

Figura 3 - Percentual de acertos de cada categoria entrevistada, dentro de cada classe.

máquinas agrícolas, os atuais envolvidos com esta atividade e os responsáveis pela sua operação e manutenção, como forma de se obter máquinas mais seguras e operações e/ou manutenções que não impliquem má utilização ou criação de fatores de riscos à saúde e/ou à integridade física de seu usuário.

Ao analisar o percentual de acertos obtido pelas categorias estudadas em cada símbolo sorteado (Figura 4), observa-se que, em símbolos bastante conhecidos pelos motoristas de automóveis, como a de "cinto de segurança”, foi obtido um grande percentual de acertos nas categorias de acadêmicos e profissionais, ficando um pouco abaixo na dos operadores/mantenedores. O fato se repete, em menor intensidade, nos símbolos “cuidado”, "afogador”, "farol principal-luz baixa”, "lavador e limpador de párabrisa" e "ventilação forçada", dando margem à confirmação da teoria de ALONÇO (2004) de que esSes símbolos, neste caso, já estão na Memória de Longo Termo (MLT), sendo portanto conservadas de forma permanente.

Constata-se também que, em todas as circunstâncias citadas anteriormente, a categoria dos operadores/mantenedores, talvez por ser uma categoria em que nem todos possuem automóveis ou acesso a eles e por não serem tão atingidos por campanhas publicitárias veiculadas pela mídia, é a que menor percentual de acertos atingiu o que, segundo ALONÇO (2004), justifica-se pelo fato de que, nesta categoria, as informações ainda estão na “Memória de Trabalho” (MT).

Por outro lado, talvez pela falta de experiência e também de efetivo conhecimento em símbolos como "avante”, "totalmente mecanizado", “variação contínua rotacional”, “variação contínua linear” e "estacionar", o rendimento dos acadêmicos é nulo. Neste caso, embora insignificante, o percentual de acertos da categoria operadores/mantenedores existe, ao contrário da categoria dos profissionais que, em alguns casos, foi nulo.

Como os símbolos apresentados no questionário foram sorteados entre os cento e um existentes na ABNT (1990), também houve a ocorrência de símbolos utilizados nos comandos e controles somente utilizados em máquinas agrícolas. Isto talvez explique o pouco conhecimento dos acadêmicos a respeito de tais símbolos e talvez indique aos cursos de Engenharia Mecânica, Engenharia Agrícola, Engenharia Florestal e Agronomia a necessidade de um fortalecimento e aprofundamento no estudo de Normas Técnicas existentes e de utilização no projeto de máquinas agrícolas, atendendo ao que foi proposto por McMAHON et al. (1997).

O fato do baixo aproveitamento dos operadores/mantenedores, de acordo com MUCKLER (1984), talvez indique a seus contratantes a necessidade de cursos de capacitação, aos representantes das concessionárias uma maior ênfase e qualidade nas entregas técnicas das máquinas e às indústrias uma padronização de acordo com a Norma Técnica dos símbolos utilizados. É preciso que todas as indústrias utilizem os mesmos símbolos e que todos possuam o mesmo significado, para, de acordo com KONTOGIANNIS (1999), oferecer sistemas de apoio ao operador de máquinas com o propósito de prevenir

Ciência Rural, v.37, n.1, jan-fev, 2007. 


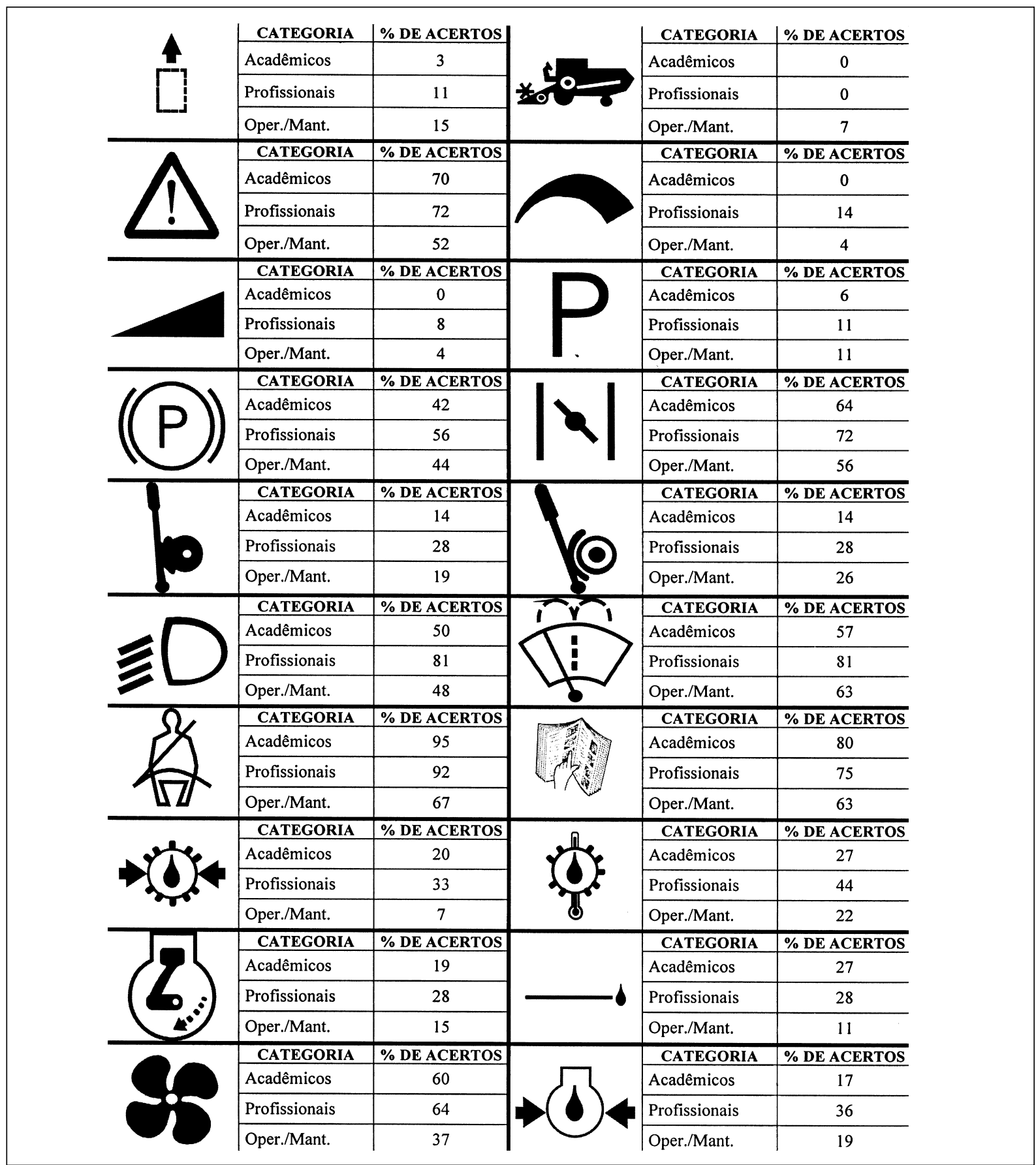

Figura 4 - Percentual de acertos em cada símbolo pelas categorias pesquisadas.

o erro humano e, com isso, reduzir o grande volume de sérios acidentes que ocorrem no meio rural.

Por fim, ao analisar o percentual de acertos da categoria dos profissionais, observa-se que, embora na maioria dos casos sejam eles os que possuem a maior margem de acertos, por serem profissionais que atuam em ensino, pesquisa, projeto e desenvolvimento na área de máquinas agrícolas, é bastante preocupante o seu baixo rendimento. Isto talvez se deva ao descaso de alguns, à falta de capacitação de outros, à inexistência de cobrança do mercado consumidor, à Norma Técnica defasada em relação aos vários comandos e controles que foram inseridos em razão da vertiginosa evolução tecnológica dos últimos quinze 
anos, ou ainda à falta de uma conscientização da necessária padronização que deve ser conferida às máquinas agrícolas produzidas no País.

\section{CONCLUSÕES}

O conhecimento sobre o significado que possuem os símbolos gráficos utilizados para caracterizar comandos e controles de máquinas agrícolas é bastante reduzido por parte dos principais envolvidos no processo. Identifica-se a urgente necessidade de este assunto ser contemplado em cursos universitários regulares ligados ao assunto, como forma de capacitar os futuros responsáveis pelo projeto de máquinas agrícolas, e também em cursos de atualização dirigidos para operadores/mantenedores e profissionais atualmente envolvidos com esta atividade, como forma de se obter máquinas mais seguras e de que sua operação e/ou manutenção que implique má utilização.

\section{AGRADECIMENTOS}

Ao professor da Universidade Federal de Santa Maria/ Centro de Ciências Rurais/ Departamento de Fitotecnia (UFSM/CCR/DF) Dr. Alessandro Dal'Col Lúcio e ao Acadêmico do Curso de Agronomia da UFSM Vilson Benz (Bolsista FIPE/ UFSM), pelas valiosas sugestões oferecidas na redação e na análise estatística deste trabalho.

\section{REFERÊNCIAS}

ALONÇO, A. dos S. Metodologia de projeto para a concepção de máquinas agrícolas seguras. 2004. $221 \mathrm{f}$. Tese (Doutorado em Eng. Mecânica) - UFSC.

AMERICAN SOCIETY OF AGRICULTURAL ENGINEERS. ASAE S304.5 - Symbols for operator controls on agricultural equipment. USA, Jun 1984. 4p.

ASSOCIAÇÃO BRASILEIRA DE NORMAS TÉCNICAS (São Paulo, SP). NBR 11379 - Símbolos gráficos para máquinas agrícolas. São Paulo, 1990. 13p.

CARPES JÚNIOR, W.P. Análise da segurança humana para desenvolvimento de produtos mais seguros. 2001. 251f. Tese (Doutorado em Engenharia de Produção) Universidade Federal de Santa Catarina.
FIALHO, F.A.P. Uma introdução à engenharia do conhecimento. Florianópolis: UFSC/PPGEP, 2000. np. (Caderno Didático).

FRESE, M. Error management or error prevention: two strategies to deal with errors in software design. In: BULLINGER, H.J. (Ed.). Human aspects in computing: design and use of interactive systems. Amsterdam: Elsevier, 1991. p.776782. Acesso em 9 abr. 2003. On line. Disponível na Internet em: <http://www.elsevier.com/locate/dsw>.

GIBBINGS, J.C. The systematic experiment. Cambridge: Cambridge University, 1986. 320p.

INTERNATIONAL ORGANIZATION FOR STANDARDIZATION (Geneva). ISO 3767/1. Tractors, machinery for agriculture and forestry, powered lawn and garden equipment Symbols for operator controls and other displays - Part 1: Common symbols. Geneva, 1982. 5p.

INTERNATIONAL ORGANIZATION FOR STANDARDIZATION (Geneva). ISO 3767/2-1982 Tractors, machinery for agriculture and forestry, powered lawn and garden equipment - Symbols for operator controls and other displays - Part 2: Symbols for agricultural tractors and machinery. Geneva, 1982. 3p.

KONTOGIANNIS, T. User strategies in recovering from errors in man-machine systems. Safety Science, v.32, p.49-68, 1999. Acesso em: 10 jan. 2003. On line. Disponível na Internet em: <http://www.elsevier.com/locate/dsw>.

McMAHON, C.A. et al. A classification of errors in design. In: INTERNATIONAL CONFERENCE ON ENGINEERING DESIGN - ICED 97, 1997, Tampere. Annals... Tampere: A. Riitahuhta, 1997. V.3, p.119-124.

MUCKLER, F.A. Standards for the design of controls: a case history. Applied Ergonomics, v.1, p.175-178, 1984.

PINKER, S. Como a mente funciona. São Paulo: Companhia das Letras, 1998. 666p.

SPIEGEL, M.R. Estatística. 13.ed. São Paulo: Makron books, 1993. 643p.

ZAPF, D.; REASON, J. Human error and error handling. Applied Psychology: an international review. v.43, n.4, p.427-432, 1994. Acesso em 10 de Janeiro de 2003. On line. Disponível na Internet em: <http://www.elsevier.com/locate/dsw>. 